\title{
Simulation of droplet spreading on micro-CT reconstructed 3D real porous media using the volume-of-fluid method
}

\author{
M. Aboukhedr ${ }^{1 *}$, N. Mitroglou ${ }^{1}$, A. Georgoulas ${ }^{2}$, M. Marengo $^{2}$, K. Vogiatzaki ${ }^{2}$ \\ ${ }^{1}$ Department of Mechanical Engineering, City University of London, UK \\ ${ }^{2}$ Advanced Engineering Centre, University of Brighton, Brighton, UK \\ ${ }^{*}$ Corresponding author: mahmoud.aboukhedr.1@city.ac.uk
}

\begin{abstract}
Droplet impact on porous media has a broad range of applications such as material processing, drug delivery and ink injection etc. The simulation studies of such processes are rather limited. To represent the spreading and absorption process of the droplet on porous materials, robust numerical schemes capable of accurately representing wettability as well as capillary effects need to be established. The current work, presents one of the first studies of droplet impact on a real porous media geometry model extracted from a micro-CT scan. The process involves processing of CT image and subsequent threshold based on the structures segmentation. The porous geometry is extracted in the form of a STL (STereoLithography) model, which, with the aid of dedicated software like ANSA and SnappyHexMesh, is converted to an unstructured mesh for successful discretization of the flow domain. The solution algorithm is developed within the open source CFD toolbox OpenFOAM. The numerical framework to track the droplet interface during the impact and the absorption phases is based on previous work [1, 2]. The volume-of-fluid (VOF) method is used to capture the location of the interface, combined with additional sharpening and smoothing algorithms to minimise spurious velocities developed at the capillary dominated part of the phenomenon (droplet recession and penetration). A systematic variation of the main factors that affect this process are considered, i.e. wettability, porous size, impact velocity. To investigate the influence of porous structures on droplet spreading, the average porosity of the media is varied between $18.5 \%$ and $23.3 \%$. From these numerical experiments, we can conclude that the droplet imbibition mainly depends on the porous wettability and secondly that the recoiling phase can be observed in the hydrophobic case but not in the hydrophilic case.
\end{abstract}

Keywords; Droplet Spreading, Droplet Absorption, Porous media, 3D micro-Topography, VOF

\section{Introduction}

Micro-scale fluid phenomena are involved in various applications and research areas [3]. Understanding the behaviour of droplet spreading on porous media is important for a variety of industrial applications, such as ink jet printing, raindrops on textile, spray paint on wood, 3D-printing, penetration of rain drops into building walls, needle less injection, coating of porous materials, irrigation, cooling of electronic devices etc. Droplet spreading on solid flat surfaces has been the subject of numerous experimental and numerical studies over the last few decades $[5,6]$. However, droplet impact on porous media is still far from being understood. Studies of such micro-scale fluid phenomena need careful and combined consideration of droplet dynamics and porous media characteristics. Generally, this phenomenon is controlled by two main counter-acting processes: droplet spreading on porous surfaces and imbibition inside the porous media [8]. As the droplet spreads on the surface it also fills the voids of the porous material due to capillary action. The spreading behaviour of the impinging droplet on the surface is known to depend on the liquid properties, i.e. density, viscosity and surface tension, impact conditions such as drop size and impact velocity, and surface wettability [9]. Absorption, on the other hand, is governed by both, the liquid and the porous medium properties, like porosity, pore size and pore wettability [10]. Droplet spreading on implicit or of non-realistic shapes porous media using numerical methods has been investigated in a limited number of studies $[11,14]$. Moreover, limited CFD have been published in the literature regarding the effect of porous media heterogeneity on imbibition using explicit porous geometry. Davis and Hocking [15] models were developed to study the time evolution of drop spreading, the position of contact line and liquid motion inside the porous media. In [16] three-dimensional simulations were carried out to study the effect of impact velocity and surface roughness on the spreading of droplets on to a substrate consisting of randomly placed and orientated freely penetrating disks using the lattice-Boltzmann method. The same methodology was used in [17] to simulate the pore-level droplet spreading on a porous surface and investigated the power-law time-evolution of the wetted zone radius.

The present study attempts to numerically analyse the characteristics of a droplet interacting with a real porous surface, using a 3D porous media that is presented in [18]. A direct comparison of simulation versus experimental results found in [19] is presented, alongside an investigation of time evolution of impinging droplet shape as well as velocity. Finally, the effect of permeability is examined through the parametric study.

\section{Micro-CT porous geometry}

This section discusses procedure followed to extract a computational mesh from micro-CT scan data. As a nondestructive technique, X-ray micro-CT provides the required insight into opaque objects eliminating the need of sectioning/partitioning. A micro-CT scanner uses X-rays that penetrate a 3D object and create density-based attenuated two-dimensional projections, which are collected at small angular steps, preferably, around 360 degrees. 
These projections are later synthesised, using a variety of techniques, and the complete volume of the object is reconstructed, in terms of two-dimensional image slices. The computed stack of 2D slices usually share a resolution of a few microns and their pixel values are inextricably bound to material density-based attenuation.

Table 1. Properties of porous stones and mesh quality.

\begin{tabular}{c|cccc}
\hline & Berea B1 & Carbonate C1 & Savonnières(Exp) & Meule(Exp) \\
\hline Size $(\mathrm{mm})$ & $7.4^{3}$ & $4.5^{3}$ & $20^{3}$ & $20^{3}$ \\
Average Porosity & $18.5 \%$ & $23.3 \%$ & $26.9 \%$ & $16.6 \%$ \\
Average Pore Size (microns) & 11.9 & 20.7 & 10.3 & 9.1 \\
\hline Max aspect ratio & 11.9796 & 7.54027 & & \\
Number of cells & 5689735 & 3777008 & & \\
Max non-orthogonality (degrees) & 78.2862 & 76.1127 & & \\
\hline
\end{tabular}

Segmentation of the volume stack of X-ray micro-CT slices was performed using Retomo, a new software package from BETA CAE Systems, which is used to define the rock/air interface contours for the entire volume. The same software was used to then export the interface iso-surface as a STereoLithography (STL) type mesh with connectivity information between the generated triangles. The latter ensures a minimum (if not zero) number of free-hanging elements that could complicate the definition of computational domain. The generated surface mesh was of high quality; however, further mesh operations were conducted in the ANSA pre-processor in order to ensure that the pore and throat representations of the rock were kept intact. The produced surface was then used to define the boundary of the flow domain, which is discretized using an unstructured type mesh. During volume mesh operation, a coarse background Cartesian mesh is generated first, which is then refined around the surface boundary; this approach reduces the overall number of elements. Computational domain dimensions and meshing quality parameters are defined in Table 1. Two different specimens were used. The average pore sizes of Berea and Carbonate sandstone is 11.9 and 20.7 microns, respectively. In the present study, the numerical average porosity of media is fixed $(B 1=18.5 \%$ and $C 1=23.3 \%)$ and surface porosity varies with pore size. The computational domain was divided into two main refinement zones at the area of impact as shown in Fig. 1 (right side)
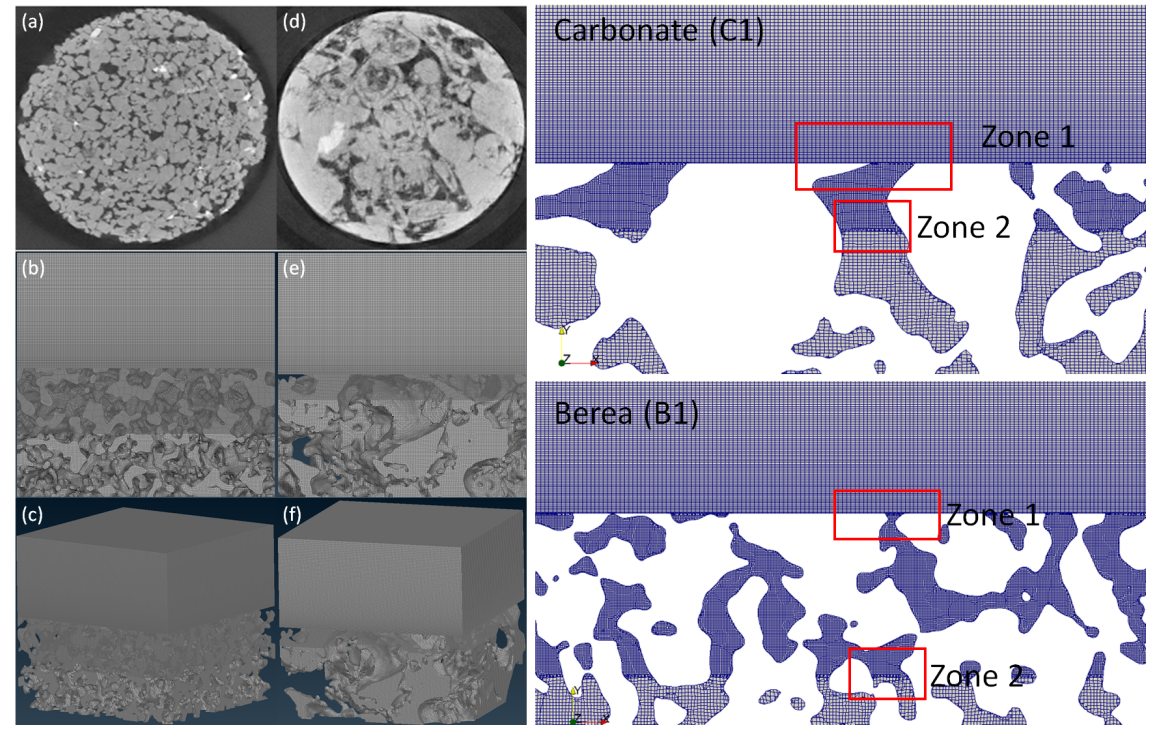

Figure 1. Numerical model for Berea (B1) and Carbonate (C1) porous media. (a,d) represent the original Mico CT for the two different porous media. ( $b$ and $c$ ) represent the computational mesh for the Bera sandstone. (e and f) represent the Carbonate.

On the right-hand side, a section inside the porous media is taken to show the mesh refinement zones

\section{Numerical set-up}

The computational model is implemented within the open source CFD toolkit OpenFOAM and is described in this section. An incompressible two-phase flow with constant phase densities $\rho_{1}$ and $\rho_{2}$ and viscosities $\mu_{1}$ and $\mu_{2}$ is considered. A single set of governing equations are solved in the entire computational domain (shared by both phases) in conjunction with a transport equation for the volume fraction of one of the phases $-\alpha-$. In the proposed numerical method, the Continuum Surface Force (CSF) description of Brackbill et al. [20] is used to represent the surface tension forces in the following form:

$$
f_{s}=\sigma \kappa \delta_{s}
$$


where the term $\kappa=-\nabla \cdot\left(\eta_{s}\right)$ represents the interface curvature, $\delta_{s}$ is a delta function concentrated on the interface and $\eta_{s}$ is the normal vector to the interface that is calculated by the following equation:

$$
\eta_{s}=\frac{\nabla \alpha_{\text {smooth }}}{\left|\nabla \alpha_{\text {smooth }}\right|}
$$

where $\alpha_{\text {smooth }}$ is the artificial smoothed indicator-function, $\delta_{s}$ is the sharpened indicator-function. In the VOF method, the indicator function $\alpha$ represents the volume fraction of one of the fluid phases in each computational cell. The indicator function evolves spatially and temporally according to an advection transport equation of the following general form:

$$
\frac{\partial \alpha}{\partial t}+\nabla \cdot(\alpha \mathbf{u})=0
$$

Ideally, the interface between both phases should be massless since it represents a sharp discontinuity. However, within VOF formulation the value of $\alpha$, at the interface, varies between 0 and 1 due to numerical diffusion. The framework described above, reflects the generalised framework of VOF methods and can be used in a large range of two-phase flow problems with various adjustments. In the following sub-sections, an enhanced version of this basic VOF framework that addresses the previously mentioned challenges, is presented in detail.

\section{Adaptive Compression Scheme (Implicit)}

To deal with the problem of numerical diffusion of $\alpha$, an extra compression term is used in OpenFOAM to control the thickness of the interface as shown below;

$$
\frac{\partial \alpha}{\partial t}+\nabla \cdot(u \alpha) \underbrace{-\nabla \cdot\left\{u_{r} \alpha((1-\alpha))\right\}}_{\text {compression term }}=0
$$

where $\left(u_{r}\right)$ is the compression velocity given by Eq. 5 . It represents the vector of relative velocity and is formulated based on the maximum velocity magnitude at the interface region and its direction, which is determined from the gradient of the phase fraction as follows:

$$
u_{r}=\min \left(C_{c} \frac{\left|\phi_{f}\right|}{|S f|}, \max \left[\frac{\left|\phi_{f}\right|}{|S f|}\right]\right)\left(\eta_{f} \cdot S_{f}\right)
$$

The term $\phi_{f}$ is the mass flux and $S f$ is the cell surface area. The compressive term is taken into consideration only at the interface region and it is calculated in the normal direction to the interface to avoid any dispersion. The maximum operation in Eq. 5 is performed over the entire domain, while the minimum operation is done locally on each face. The constant $\left(C_{c}\right)$ is a user-specified value, which serves as a parameter to obtain different cell results in different levels of compression. In most of the simulations presented here $\left(C_{c}\right)$ is taken as unity, after initial trial simulations. Higher values than unity in this case may lead to unphysical results. To make the compression methodology more general and overcome the need for a priori tuning, an adaptive algorithm has been implemented to the solver based on the following relationship:

$$
C_{a d p}=p o s\left(-\frac{U_{n} \cdot \nabla \alpha}{\left|U_{n}\right||\nabla \alpha|}, C_{\alpha}\right)
$$

where $C_{a d p}$ is the adaptive compression coefficient that acts in the volume fraction field, The constant $\left(C_{\alpha}\right)$ is a user-specified value. In our simulation $\left(C_{\alpha}\right)$ is set to be zero. The term $U_{n}$ represents each phase normal to the interface velocity. It is expressed as;

$$
U_{n}=\left(U \cdot n_{s}\right) *\left(n_{s}\right) * \operatorname{pos}(\alpha-0.01) * \operatorname{pos}(0.99-\alpha)
$$

The concept of using $U_{n}$ is shown in Fig. 2. When the profile of the colour function becomes diffusive $C_{a d p}$ value is increased in order to sharpen the interface relevant to the local diffusion field. When the profile is already sharp and additional compression is not necessary, $C_{a d p}$ will go to zero.

\section{Numerical Treatment of the Indicator Function}

After solving Eq. 4 with the additional compression term, the indicator function is updated at the cell centres. Afterwards the indicator function is obtained at the cell boundaries using a linear extrapolation from the cell centres in order to proceed with the calculation of $\eta$ and $k$. At this stage the value of the indicator function sharply changes over a thin region (as a result of the compression step). This abrupt change of the indicator function creates errors in calculating the normal vectors and the curvature of the interface, which will be used to evaluate the interfacial forces. These errors induce non-physical parasitic currents in the interfacial region. A commonly followed approach to the literature to suppress these artifacts is to compute the interface curvature from a smoothed function $\alpha_{\text {smooth }}$, which is calculated by the smoother proposed by Georgoulas et al. [22]. It should be noticed that the number of 

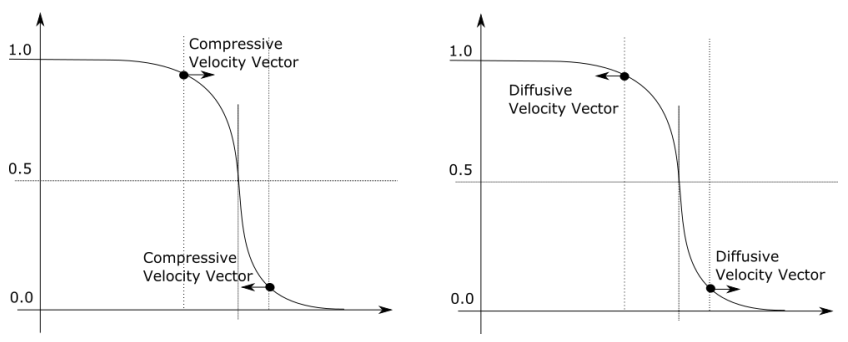

Figure 2. Schematic to represent the adaptive compression $C_{A d p}$ selection criteria

Table 2. Properties of water droplet and impact conditions for drop test.

\begin{tabular}{c|c|c|c|c}
\hline & $\rho\left(K g / m^{3}\right)$ & $\mu(K g / m s)$ & $\sigma(N / m)$ & \\
\hline Water Properties at $25^{\circ} \mathrm{C}$ & 998 & 0.001 & 0.07 & \\
\hline & $R_{0}(\mathrm{~mm})$ & $V_{i}(\mathrm{~m} / \mathrm{s})$ & We & $\operatorname{Re}$ \\
\hline Impact conditions & 1 & $0.5-1$ & $10-20$ & $498-1000$ \\
\hline
\end{tabular}

times that the proposed filter is applied should be handled with care in the calculations since there is a fine balance between smoothing the interface" and altering the curvature, especially in high curvature interfacial regions. For the presented simulations based on sensitivity analysis we repeat the smoothing loop 4 times. Following, capillary forces are calculated at the face centres based on Eq. 1. For the calculation of curvature $k$ we used the smoothing operation as described above. However special care needs to be taken for the calculation interface delta function $\delta_{s}$. In order to maintain its sharpness we also introduce a sharp function $\left(\alpha_{\text {sharp }}\right)$ modelling the capillary pressure in the case of flows through porous media. Then $\delta_{s}$ is calculated based on the $\alpha_{\text {sharp }}$ rather than the $\alpha_{\text {smooth }}$. In the final step non-physical velocities that are parallel to the fluid interface are filtered, so that the term $f_{c}-\nabla p_{c}$ that appears in the Eq 8 finally converges to zero.

\section{Capillary Pressure Jump Modelling and Filtering Numerical Errors}

At the pore scale, the momentum balance equation for incompressible fluids is used to relate the pressure gradient, inertial, capillary, gravity and viscous forces. Knowing the pressure gradient, capillary and gravity forces, the momentum balance equation can be used to update the velocity field at any time-step;

$$
\frac{D}{D t}(\rho u)-\nabla \cdot T=-\nabla p_{d}+\rho g+f_{s}-\nabla p_{c}
$$

where, $\rho g$ is the gravity force and $f_{s}$ is the capillary force. $p_{d}$ is the dynamic pressure and it reads: $p_{d}=p-p_{c}$. where $\mathrm{p}$ is the total (physical) pressure and $\mathrm{pc}$ is a potential field called the microscopic capillary pressure. $P_{c}$ is obtained from the solution of the Poisson's equation:

$$
\nabla \cdot \nabla P_{c}=\nabla \cdot f_{s}
$$

This approach includes explicitly the effect of capillary forces in the Navier-Stokes equations, and therefore allows for the filtering of the numerical errors related to the inaccurate calculation of $f_{c}$. The non-physical velocities that are parallel to the fluid interface are filtered, so that the term $f_{c}-\nabla p_{c}$ that appears in the Eq 8 finally converges to zero as discussed in [2].

\section{Wettability Modelling}

For the present calculations we implemented the Kistler model [24] in the standard and developed solver in order to be able to accurately simulate the contact line movement. The Kistler model calculates the dynamic contact angle, using the Hoffman function, $f_{H o f}$, as $\Theta_{d y n}=f_{H o f}\left[C a+f_{H o f}^{-1}\left(\Theta_{e}\right)\right]$ where $\Theta_{e}$ is the equilibrium contact angle and $\mathrm{Ca}$ the capillary number calculated based on an imposed spreading velocity.

\section{Case set-up}

Three-dimensional simulations were performed. The computational domain, the grid as well as the applied boundary conditions are shown in Fig. 3. The initial conditions for the simulated geometry are illustrated in Fig. 3, while the material properties and the initial conditions for the impacting liquid (water) are summarised in Table 2. Different values for the wetting conditions are illustrated in Table 3, where ten numerical simulations were performed to show the influence of wetting on spreading behaviour at different porous media. In each case, initial droplet radius is set to $\mathrm{R}=1 \mathrm{~mm}$. At the first time step, a spherical liquid drop is patched inside the numerical domain in such a way where the liquid phase just on top the porous surface. 


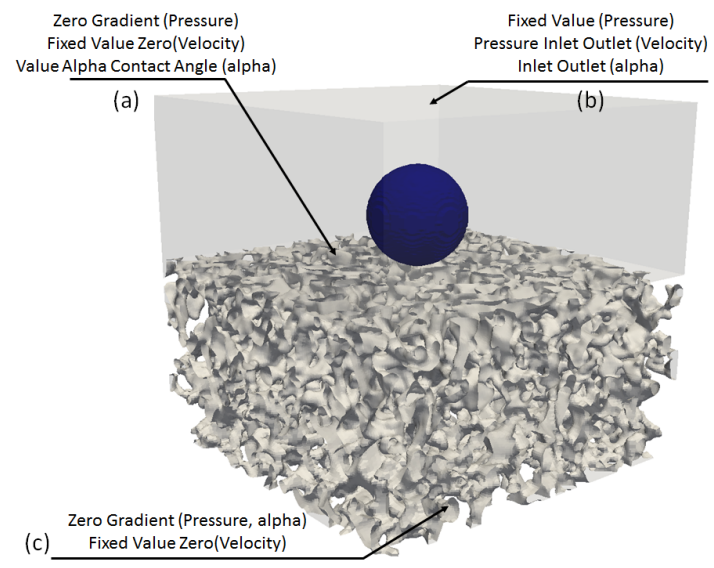

Figure 3. Three-dimensional computational domain, mesh and boundary conditions

\section{Results and discussion}

In the following sections, numerical simulations are presented for one case that assesses the performance of the proposed model. Then the influence of porous wettability on spreading radius dynamics using two different porous media is investigated. Also, the droplet penetration and effect of wettability on inertial spreading stage and the viscous spreading stage are observed. Calculations with the standard VOF-based solver of OpenFOAM (interFoam) are also included for comparison purposes. Additionally, the droplet radius at the time of impact was measured using a custom Matlab code to enable comparison of the results to the given experimental data.

\section{Validation of numerical method}

The test case used to validate the numerical model is of a droplet impacting a Berea sandstone segment with impact velocity $V_{i}=1 \mathrm{~m} / \mathrm{s}(\mathrm{We}=20, \mathrm{Re}=1000)$. The numerical simulation is compared against experiments reported in $[8,19]$. The experimental porous media namely -Meule- is described in Table 1. Although our numerical model and the one that experimental data are taken are not the same materials (they do not share the same porous structures) they share similar average porosity and average pore sizes. Depending on how close are predictions with experimental data, conclusions can be extracted regarding the importance of these two parameters (porosity and average pore sizes) to the evolution of the spreading. One Should note that careful treatment should be taken while modelling the wetting conditions as they may dominate the spreading behaviour. As reported in the experiments $[8,19]$ the advancing and receding contact angles are $\left(125^{\circ}\right.$ and $\left.25^{\circ}\right)$, which is used as initial conditions for our Kistler wetting model in order to guarantee that the numerical and experimental model have the same wetting behavior. Figure 4 shows the normalised time evolution $(t / \tau)$ of the droplet spreading $(r / R)$, where $\mathrm{R}$ is the initial droplet radius, and $r$ is the calculated droplet radius over the porous media during spreading. Spreading radius $(r)$ over the porous media is found to be irregular compared to the normal droplet spreading over a flat surface (not a perfect circular shape). To determine the spreading radius ( $r$ ), we use our numerical data to calculate the wetted area of the irregular spreading shape, and extract an equivalent radius based on the centre of gravity, as seen in Fig. 4, where the black and white picture represents the droplet shape over the porous media while the blue line represents the fitted circle determined from post-processing. The normalised time is defined as the inertial time scale of the first stage of wetting, where $\tau=\left(\frac{\rho R^{3}}{\sigma}\right)^{0.5}$ [25]. As we can see the phenomenon -based on the experimental data for the droplet diameter- is characterised by the following phases: Initially, the droplet diameter is increased (spreading phase) reaching to a maximum length and then stabilises. Although for the first phase the same increase is noticed in the phenomena of droplet spreading over solid surfaces, the second part is different. When droplet impacts on a porous medium we can notice that the diameter remains almost constant implying that the imbibition phase has started (for this case at $(t / \tau)=0.6$ ) [26]. These phases are well captured by our new solver (interPore) while the standard solver fails to predict the correct transition to the imbibition phase. Also, it can be seen that for the imbibition phase our code (interPore) has a very close match with the experiments. It is also an indication that porosity and average pore diameter are the two most important controlling parameters since as mentioned above simulations and experimental data share porosity and average pore diameter but not pore structure. Finally, we notice that for the spreading phase there are some differences between experimental and numerical curve indicating a slower spreading process of the droplet of the numerical model in comparison to experimental one. This can potentially indicate that average pore size might not be enough to describe the spreading process and more information for the pore distribution are necessary. Using the Kistler boundary condition is a key element in capturing the correct physical behaviour for the droplet spending. Nevertheless, by imposing the Kistler boundary condition to the standard solver (interFoam), we noticed an overestimation for droplet size during the spreading phase. The reason comes from the wrong calculated contact line velocity during the inertial phase caused by the parasitic currents. The overestimated spreading is not reflected in our simulation using the modified solver (interPore) due to the parasitic current filter used. Based on [25] we also plotted the theoretical power-law $(r / R)$ as 


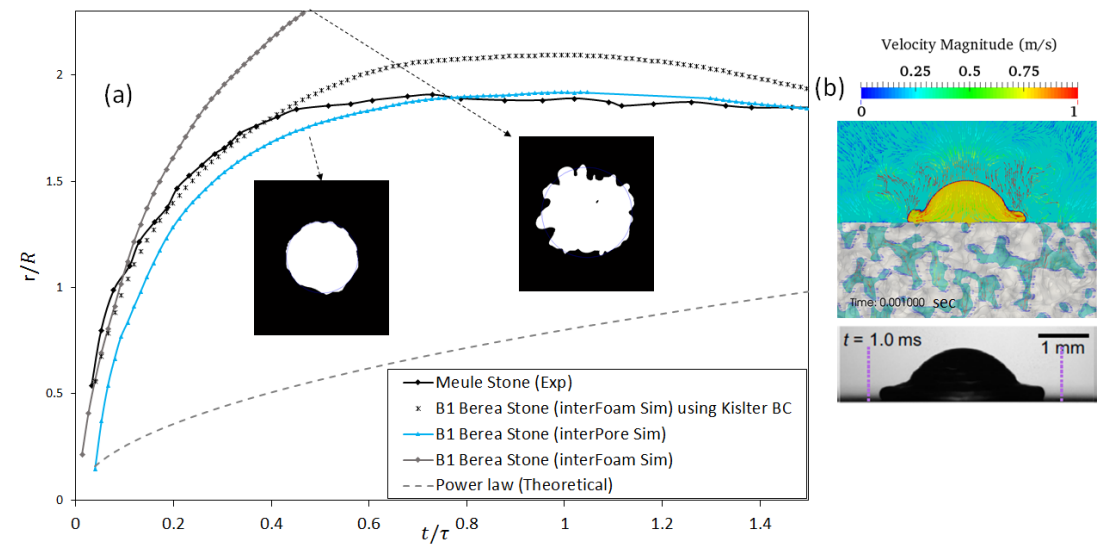

Figure 4. Comparison of drop impacts for $D_{0}=2.0 \mathrm{~mm}$ and $V_{i}=1.0 \mathrm{~m} / \mathrm{s}$ on Berea stone (numerical) and Meule stone (experimental). (a) is a normalised wetted zone width $\mathrm{r} / \mathrm{R}$ evolution for water droplet at $\mathrm{We}=28.5$. Developed solver (interPore) at wetting conditions of $\theta_{A}=125^{\circ}, \theta_{R}=25^{\circ}$ at the porous surface and porous media, Standard solver (interFoam) at wetting conditions of $\theta_{e q}=25^{\circ}$ at the porous surface and porous media. (b) Snapshots of drop impact on porous materials at time $=1$ ms

$\frac{r}{R}=0.8\left(\frac{t}{\tau}\right)^{0.5}$. The power law fails to estimate the correct spreading behaviour due to the unaccounted influence of the porous structure and wettability effect.

\section{Influence of porous wettability on radius dynamics}

To provide further insight into the local phenomena which drive capillary imbibition of the droplet inside porous media, two different material are presented here with different wetting behaviour The contact angle values used to observe the influence of wettability and porosity are shown in Table 3. Figure (5) shows the droplet behaviour (represented by the iso-surface of the droplet volume fraction at 0.5 ) at two different porous wetting conditions (hydrophobic porous surfaces and hydrophilic porous surfaces) for two different porous media (Carbonate and Berea). Two features can be identified in both porous cases; firstly, the droplet invasion mainly depends on the porous wettability regardless the change of the porosity. Secondly, the recoiling phase can be observed in the hydrophobic case but not in the hydrophilic case for both porous structures. At hydrophobic conditions of the pore shows less liquid absorption with a recoiling phase, yet it is still characterised by pinning behaviour. At the hydrophilic porous wetting, regardless of imposing a dynamic wetting conditions on the porous surface in our simulations, no recoiling phase is observed. However, the droplet edge is seen to undergo same pinning with much more absorption, behaviour which limits its spreading. Spreading on the solid surfaces between the holes depend mainly on the pore wettability for same solid surface wetting conditions. The interface shape was captured over time as shown in Fig. 5. The hydrophobic case shows small liquid fingers in the initial phase, which tends to be receding from the pore space again during the recoiling phase. While for the hydrophilic cases, the fingering effect after the invasion and during the initial phase tends to increase by time. This leads to a decrease in droplet size in the recoil phase, yet keeping same spreading diameter. Figure 6 shows the normalised droplet spreading at two different porosities and a wide range of pore wettability. For Figure 6a Case 1 and Case 2 undergo same surface spreading with different penetration behaviour. While Case 3 shows mush less penetration associated with surface spreading. While $6 \mathrm{~b}$ Case 6 and Case 8 undergo maximum penetration duo to the high porosity value. Case 8 shows an increase in surface spreading similar to Case 4.

\section{Conclusions}

In this work, a framework initially developed to capture droplet interface dynamics at low capillary numbers is extended in order to be used in the study of the impact of liquid droplets on a real porous material reconstructed

\begin{tabular}{c|cccc}
\hline Case & Porous model & Surface contact angle & Pore Contact Angle & Vel $(\mathrm{m} / \mathrm{s})$ \\
\hline Case 1 & B1 & $\theta_{A}=125^{\circ}, \theta_{R}=25^{\circ}$ & $\theta_{A}=125^{\circ}, \theta_{R}=25^{\circ}$ & 0.5 \\
Case 2 & B1 & $\theta_{A}=125^{\circ}, \theta_{R}=25^{\circ}$ & $\theta_{e q}=0^{\circ}$ & 0.5 \\
Case 3 & B1 & $\theta_{A}=125^{\circ}, \theta_{R}=25^{\circ}$ & $\theta_{e q}=180^{\circ}$ & 0.5 \\
Case 4 & B1 & $\theta_{A}=60^{\circ}, \theta_{R}=22^{\circ}$ & $\theta_{A}=60^{\circ}, \theta_{R}=22^{\circ}$ & 0.5 \\
Case 5 & C1 & $\theta_{A}=125^{\circ}, \theta_{R}=25^{\circ}$ & $\theta_{A}=125^{\circ}, \theta_{R}=25^{\circ}$ & 0.5 \\
Case 6 & C1 & $\theta_{A}=125^{\circ}, \theta_{R}=25^{\circ}$ & $\theta_{e q}=0^{\circ}$ & 0.5 \\
Case 7 & C1 & $\theta_{A}=125^{\circ}, \theta_{R}=25^{\circ}$ & $\theta_{e q}=180^{\circ}$ & 0.5 \\
Case 8 & C1 & $\theta_{A}=60^{\circ}, \theta_{R}=22^{\circ}$ & $\theta_{A}=60^{\circ}, \theta_{R}=22^{\circ}$ & 0.5 \\
\hline
\end{tabular}

Table 3. Imposed contact angle for porous media surface (using Kistler, dynamic contact angle model) with different assumptions concerning the contact angle of the pore surfaces (varies from fully hydrophilic to fully hydrophobic) 


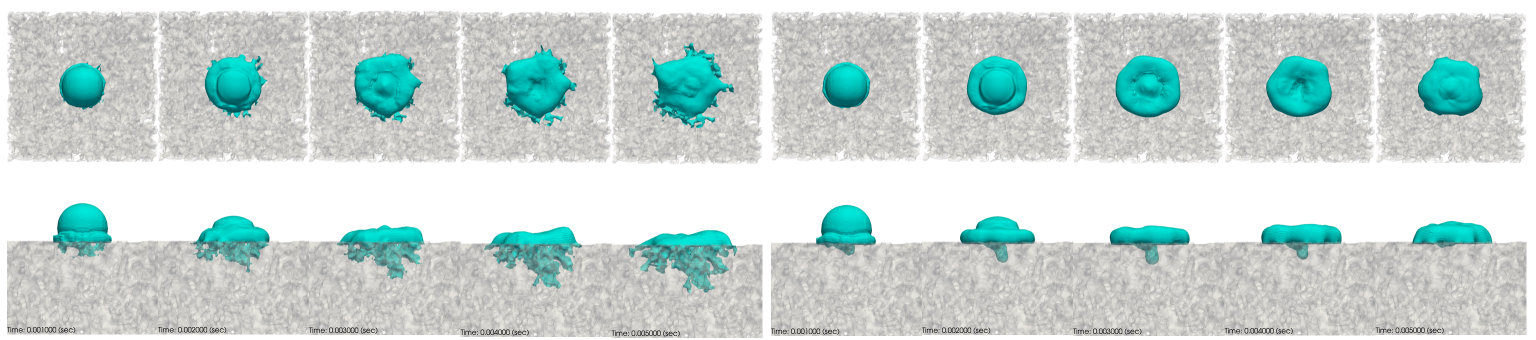

(a) Case 2 represents fully hydrophobic conditions for Berea (b) Case 3 represents fully hydrophilic conditions for Berea sandstone (B1)

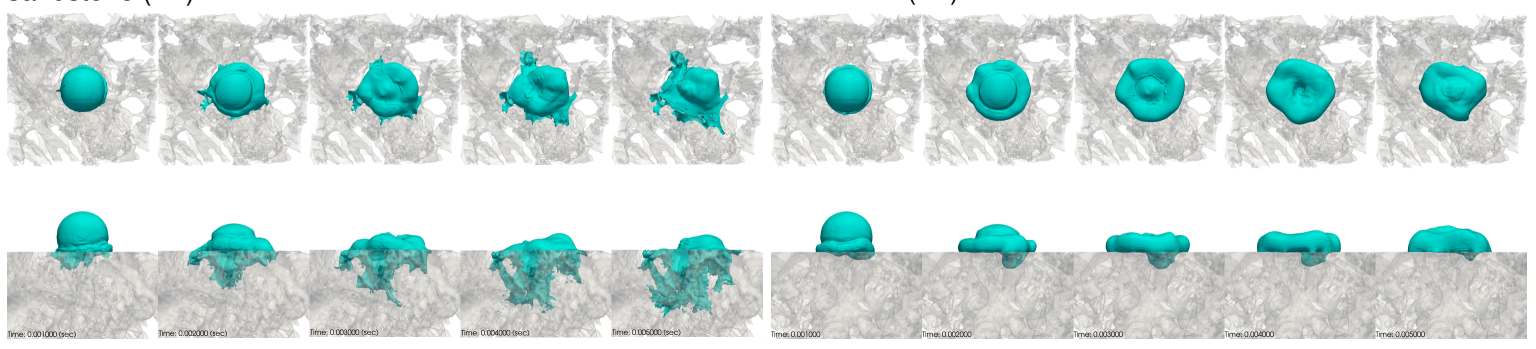

(c) Case 6 represents fully hydrophobic conditions for Carbon- (d) Case 7 represents fully hydrophilic conditions for Carbonate $(\mathrm{C} 1)$ ate $(\mathrm{C} 1)$

Figure 5. The early stage of capillary imbibition of pore after Droplet impacting the solid surfaces. Four views are provided at each five-time steps. The figures show two different wetting conditions ranges from fully hydrophobic to fully hydrophilic.

from micro-CT scanned pictures. We initially test our framework against experimental data from the Meule stone. The Meule stone and the material used for our simulations share the same porosity and average pore size but not the same pore structure. We observe that regardless of this difference both spreading and imbibition phase are well captured by the model which indicates a) that our framework is well suited to represent a complicated phenomenon like this and $b$ ) that the phenomenon as such is governed only by the two parameters. Moreover, we extend our study to the effect of wettability. Two different porous wetting conditions (hydrophobic porous surfaces and hydrophilic porous surfaces) for two different porous media (Carbonate and Berea). From these numerical experiments, it can be concluded that the droplet imbibition mainly depends on the porous wettability regardless the change of the porosity and secondly that the recoiling phase can be observed in the hydrophobic case but not in the hydrophilic case.

\section{References}

[1] M. Abokhedr, K. Vogiatzaki, N. Mitroglou, and E. Gavaises. A numerical simulation of single and two-phase flow in porous media: A pore sale observation of effective microscopic forces. 6th BETA CAE International Conference, 2, 2015.

[2] M Aboukhedr, M Gavaises, A Georgoulas, M Marengo, and K Vogiatzaki. Numerical investigation of droplet spreading on porous and non-porous surfaces. ILASS Europe, 27th Annual Conference on Liquid Atomization and Spray Systems, Brighton, UK, 2016.

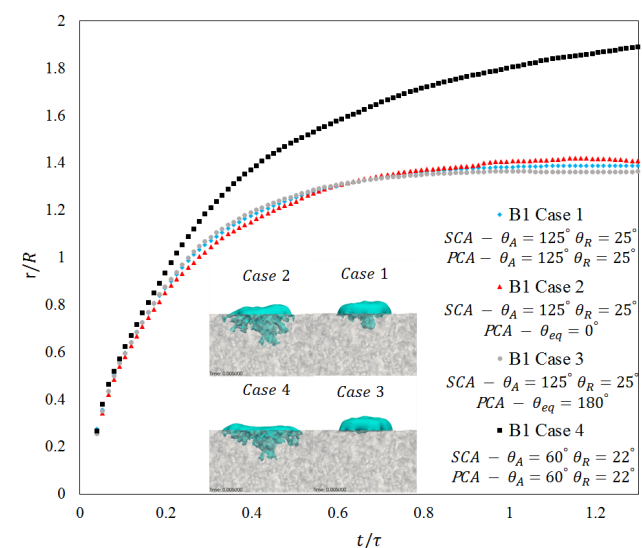

(a) B1

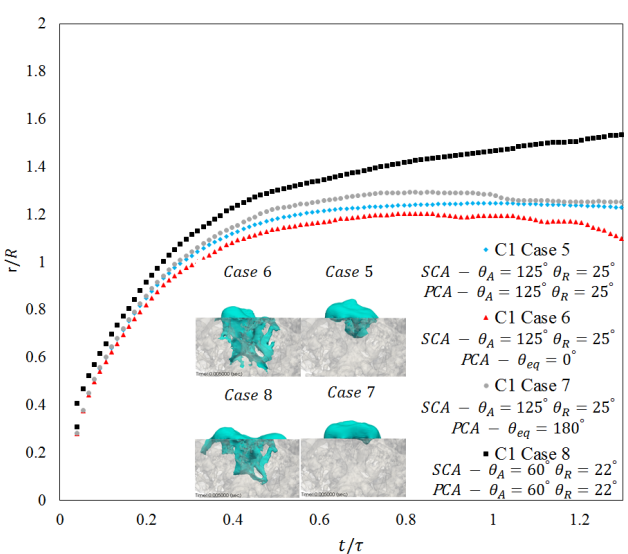

(b) $\mathrm{C} 1$

Figure 6. Normalised radius $r / R$ with different surface and pore contact angle vs. normalised time $t / \tau$ for two different porous media Carbonate (C1) and Berea sandstone (B1). Supporting images are for time $t=0.005$ (sec) 
[3] Tatiana Gambaryan-Roisman. Liquids on porous layers: wetting, imbibition and transport processes. Current Opinion in Colloid \& Interface Science, 19(4):320-335, 2014.

[4] Š Šikalo, H-D Wilhelm, IV Roisman, S Jakirlić, and C Tropea. Dynamic contact angle of spreading droplets: Experiments and simulations. Physics of Fluids, 17(6):062103, 2005.

[5] Kensuke Yokoi, Damien Vadillo, John Hinch, and lan Hutchings. Numerical studies of the influence of the dynamic contact angle on a droplet impacting on a dry surface. Physics of Fluids, 21(7):072102, 2009.

[6] R Rioboo, M Marengo, and C Tropea. Time evolution of liquid drop impact onto solid, dry surfaces. Experiments in Fluids, 33(1):112-124, 2002.

[7] Martin Rein. Phenomena of liquid drop impact on solid and liquid surfaces. Fluid Dynamics Research, 12(2): 61-93, 1993.

[8] JB Lee, AI Radu, P Vontobel, D Derome, and J Carmeliet. Absorption of impinging water droplet in porous stones. Journal of colloid and interface science, 471:59-70, 2016.

[9] AL Yarin. Drop impact dynamics: splashing, spreading, receding, bouncing.... Annu. Rev. Fluid Mech., 38: 159-192, 2006.

[10] Karen P Hapgood, James D Litster, Simon R Biggs, and Tony Howes. Drop penetration into porous powder beds. Journal of Colloid and Interface Science, 253(2):353-366, 2002.

[11] Homayun K Navaz, Bojan Markicevic, Ali R Zand, Yuri Sikorski, Ewen Chan, Matthew Sanders, and Terrence G D'Onofrio. Sessile droplet spread into porous substrates-determination of capillary pressure using a continuum approach. Journal of colloid and interface science, 325(2):440-446, 2008.

[12] NC Reis, RF Griffiths, MD Mantle, and LF Gladden. Investigation of the evaporation of embedded liquid droplets from porous surfaces using magnetic resonance imaging. International Journal of Heat and Mass Transfer, 46 (7):1279-1292, 2003.

[13] Neyval C Reis, Richard F Griffiths, and Jane M Santos. Numerical simulation of the impact of liquid droplets on porous surfaces. Journal of Computational Physics, 198(2):747-770, 2004.

[14] Neyval C Reis, Richard F Griffiths, and Jane Méri Santos. Parametric study of liquid droplets impinging on porous surfaces. Applied Mathematical Modelling, 32(3):341-361, 2008.

[15] SH Davis and LM Hocking. Spreading and imbibition of viscous liquid on a porous base. Physics of Fluids (1994-present), 11(1):48-57, 1999.

[16] VM Starov, SA Zhdanov, and MG Velarde. Spreading of liquid drops over thick porous layers: complete wetting case. Langmuir, 18(25):9744-9750, 2002.

[17] X Frank and Patrick Perre. Droplet spreading on a porous surface: A lattice boltzmann study. Physics of Fluids (1994-present), 24(4):042101, 2012.

[18] Hu Dong and Martin J Blunt. Pore-network extraction from micro-computerized-tomography images. Physical review E, 80(3):036307, 2009.

[19] JB Lee, D Derome, and J Carmeliet. Drop impact on natural porous stones. Journal of colloid and interface science, 469:147-156, 2016.

[20] J.U Brackbill, D.B Kothe, and C Zemach. A continuum method for modeling surface tension. Journal of Computational Physics, 100(2):335 - 354, 1992. .

[21] B. Lafaurie, C. Nardone, R. Scardovelli, S. Zaleski, and G. Zanetti. Modelling merging and fragmentation in multiphase flows with surfer. Journal of Computational Physics, 113(1):134 - 147, 1994. .

[22] A. Georgoulas, P. Koukouvinis, M. Gavaises, and M. Marengo. Numerical investigation of quasi-static bubble growth and detachment from submerged orifices in isothermal liquid pools: The effect of varying fluid properties and gravity levels. International Journal of Multiphase Flow, 74:59 - 78, 2015.

[23] A. Q. Raeini, M. J. Blunt, and B. Bijeljic. Modelling two-phase flow in porous media at the pore scale using the volume-of-fluid method. Journal of Computational Physics, 231(17):5653 - 5668, 2012.

[24] S.F. Kistler. Hydrodynamics of wetting. Marcel Dekker, New York, 1993.

[25] Anne-Laure Biance, Christophe Clanet, and David Quéré. First steps in the spreading of a liquid droplet. Physical Review E, 69(1):016301, 2004.

[26] J.B. Lee, A.I. Radu, P. Vontobel, D. Derome, and J. Carmeliet. Absorption of impinging water droplet in porous stones. Journal of Colloid and Interface Science, 471:59 - 70, 2016. ISSN 0021-9797. . 